\title{
Bayesian Fault Diagnosis Using Process Knowledge of Response Information
}

\author{
Wenbing Zhu', a , Ruohan Chen ${ }^{1, b}$ and Sun Zhou ${ }^{1, c^{*}}$ \\ ${ }^{1}$ Department of Automation, Xiamen University, 361005 P.R.China \\ âwbzu@stu.xmu.edu.cn, b373464877@qq.com, 'zhousun@xmu.edu.cn
}

\begin{abstract}
Keywords: Fault detection and diagnosis, Bayesian inference, response information
\end{abstract}
Abstract. Process fault diagnosis is a topic of significant practical interest. Bayesian fault diagnosis methods have been developed to identify the problem source from all monitors of the process. However in a large scale industrial process, taking all the monitors into account not only increases computation burdens but also leads to spurious diagnosis. This paper proposes a new approach to obtain a more reliable diagnosis under Bayesian frame. It explicitly takes the process knowledge expressed as response matrix into consideration to estimate the likelihood in Bayesian inference. The simulation demonstrates that the proposed approach is able to improve the diagnosis even when some abnormal mode data is sparse or not available in the historical dataset.

\section{Introduction}

In process industries, fault detection and diagnosis (FDD) is a topic with practical significance. Its purpose is to identify and isolate in the process the components that have failed [1]. FDD methods can be generally divided into model-based and data-driven methods [2-4]. While for complex systems it is generally difficult to establish first principle process models, and it is reported that knowledge-based models, like causal models, etc. may lead to spurious results [3], data-driven approaches utilize process history data instead of process models. A Bayesian frame for control loop diagnosis has been proposed since 2008 [5]. It takes all monitors into consideration and synthesizes them to isolate the fault source through Bayesian inference. However in large scale process there may be dozens of or even more than a hundred monitors. To take into account all the monitors not only increases computation burdens but also lead to spurious diagnosis. This paper proposes a new approach to obtain a more reliable diagnosis by combining the process background knowledge under Bayesian frame. The proposed approach is demonstrated by the simulation to be able to improve the fault diagnosis even when historical data for some modes are not available.

\section{Bayesian Inference Using Response Information}

Problem Formulation. Suppose besides the normal mode, some abnormal modes, each denoted as $m_{j}$, $j \in\{1, \ldots, J\}$ where $J$ is the number of all possible modes under consideration, may arise due to some faults or disturbances in the process operating. Some sensors or algorithms are designed to monitor the process performance. An observation of the monitor readings is denoted as $E$, a vector variable including all $R$ monitor readings. One such observation together with its underlying mode composes one sample. The task is given the current observation $E=e_{i}$, historical dataset, $D$, and all other information at hand, $i_{R}$, like the response information, to determine the underlying process fault mode. In Bayesian frame the task is to compute the posterior probability of all possible modes

$$
p\left(M=m_{j} \mid E=e_{i}, D, i_{R}\right) .
$$

Response Information. Process knowledge, $i_{R}$, concerns the relationship between monitor readings and process modes. It is about that some monitor readings are not affected by certain modes, but remain the same distribution as under $N F$ mode. This kind of information is called Response Information as it characterize whether some readings may respond to certain modes. This information can be obtained from the process knowledge such as the physical flowchart or the construction of 
monitoring functions that are used as observations, etc. For instance, if under an abnormal mode there is a monitor remaining the same distribution as under the normal process mode, this monitor can be regarded as independent of that abnormal mode. Response information can be expressed as a matrix. Table 1 gives an example.

Table 1 Example of a Response matrix

\begin{tabular}{llllll}
\hline & $m_{1}$ & $m_{2}$ & $m_{3}$ & $m_{4}$ & $m_{5}$ \\
\hline \hline$E_{1}$ & $*$ & $*$ & 0 & 0 & $*$ \\
\hline$E_{2}$ & $*$ & $*$ & 0 & 0 & $*$ \\
\hline$E_{3}$ & 0 & $*$ & 0 & $*$ & 0 \\
\hline$E_{4}$ & $*$ & 0 & $*$ & $*$ & $*$ \\
\hline$E_{5}$ & 0 & $*$ & $*$ & $*$ & $*$ \\
\hline
\end{tabular}

In this matrix, a 0 in the $j^{\text {th }}$ column and $k^{\text {th }}$ row represents that the $k^{\text {th }}$ monitor is not affected by the $j^{\text {th }}$ mode, while a ${ }^{*}$ denotes that the corresponding reading has some chance to be affected by this mode.

Bayesian Inference. From the response matrix the monitor sets, $O_{m_{j}}$, affcted by mode $m_{j}$ which is corresponding to $*$ in the response matrix can be obtained. The element index set of $O_{m_{j}}$ is $I_{m_{j}}=\left\{i, E_{i} \in O_{m_{j}}, i \in\{1, \mathrm{~K}, R\}\right\}$. Suppose its element number is $I$. Similarly, the complementary set of $O_{m_{j}}, O_{\bar{m}_{j}}$, is the observation element set that is not affected by $m_{j}$, which is corresponding to 0 in the response matrix. And its element number is $R-I$.

Hence the observation vector variable can be divided into two subparts, one is readings of monitors dependent on mode $m_{j}$, denoted as $E_{m_{j}}$, and the other is monitors independent of $m_{j}, E_{\bar{m}_{j}}$.

$$
E=\left(E_{1}, \mathrm{~K}, E_{R}\right)=\left(E_{I_{m_{j}}[1]}, \mathrm{K}, E_{I_{m_{j}}[I]}, E_{I_{\bar{m}_{j}}[1]}, \mathrm{K}, E_{I_{\bar{m}_{j}}[R-I]}\right)=\left(E_{m_{j}}, E_{\bar{m}_{j}}\right) \text {. }
$$

So $E=e_{i}$ can be expressed as $\left(E_{m_{j}}, E_{\bar{m}_{j}}\right)=\left(e_{m_{j}, p}, e_{\bar{m}_{j}, q}\right)$ where $e_{m_{j}, p}$ and $e_{\bar{m}_{j}, q}$ are one realization of $E_{m_{j}}$ and $E_{\bar{m}_{j}}$, respectively.

Note that $E_{\bar{m}_{j}}$ is independent of mode $m_{j}$ and apply Bayes' theorem to obtain the posterior probability stated as Eq. (1).

$$
\begin{aligned}
& p\left(M=m_{j} \mid E=e_{i}, D, i_{R}\right) \\
= & p\left(M=m_{j} \mid\left(E_{m_{j}}, E_{\bar{m}_{j}}\right)=\left(e_{m_{j}, p}, e_{\bar{m}_{j}, q}\right), D, i_{R}\right) \\
= & p\left(M=m_{j} \mid E_{m_{j}}=e_{m_{j}, p}, D, i_{R}\right) \\
\propto & p\left(E_{m_{j}}=e_{m_{j}, p} \mid M=m_{j}, D, i_{R}\right) \times p\left(M=m_{j} \mid D, i_{R}\right) .
\end{aligned}
$$

The first factor on the right side is a likelihood probability of the current observation given a possible mode, and the second factor is the prior probability of mode $m_{j}$, given historical dataset $D$ and the background knowledge $i_{R}$ expressed as response information.

The likelihood can be computed by marginalizing over all realizations of the unaffected observation variable $E_{\bar{m}_{j}}$.

$$
p\left(E_{m_{j}}=e_{m_{j}, p} \mid M=m_{j}, D, i_{R}\right)=\sum_{t} p\left(E_{m_{j}}=e_{m_{j}, p}, E_{\bar{m}_{j}}=e_{\bar{m}_{j}, t} \mid M=m_{j}, D, i_{R}\right) .
$$


To compute each term in the sum equation, introduce parameters $\Theta_{m_{j}}$ with values $\theta_{m_{j}}=\left(\theta_{1 m_{j}}, \mathrm{~K}, \theta_{K m_{j}}\right)$ such that $p\left(E=e_{k} \mid M=m_{j}, \Theta_{m_{j}}=\theta_{m_{j}}\right)=\theta_{k m_{j}}$.

It is reasonable to let $f_{\Theta}(\theta)$ be Dirichlet distributed, i.e.

$$
f_{\Theta}(\theta)=\frac{\Gamma\left(\sum_{m=1}^{M} \alpha_{m}\right)}{\prod_{m=1}^{M} \Gamma\left(\alpha_{m}\right)} \prod_{m=1}^{M} \theta_{m}^{\alpha_{m}-1}, \alpha_{m}>0
$$

where $\Gamma(\cdot)$ is the gamma function with given parameters $\alpha=\left(\alpha_{1}, \mathrm{~K}, \alpha_{M}\right)$. Let $n_{m}$ be the count of samples where $E=e_{m}$, and let $N$ and $A$ be the sum of $n_{m}$ and $\alpha_{m}$, respectively. Then following the Bayesian diagnosis inference it holds that the likelihood

$$
p\left(E_{m_{j}}=e_{m_{j}, p} \mid M=m_{j}, D, i_{R}\right)=\frac{\sum n_{e_{m_{j}, p}, e_{\bar{m}_{j}, t}, m_{j}}+\sum_{t} \alpha_{e_{m_{j}, p}, e_{\bar{m}_{j}, t}, m_{j}}}{N_{m_{j}}+A_{m_{j}}} .
$$

Therefore by using Eq. (3), (6), we obtain the posterior probability of each possible mode. Then the mode with the maximum probability will be diagnosis as the current mode of the process.

\section{Simulation}

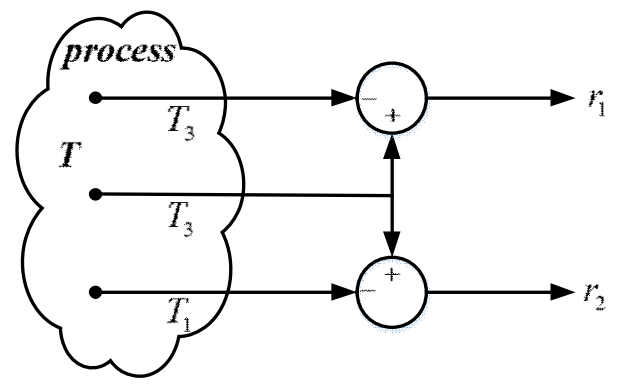

Figure 1 A sample process with three temperature measurements.

A sample process as shown in Fig. 1 is used to test the proposed approach. Three residual monitors $r_{1}, r_{2}, r_{3}$ are formed using the temperature measurements. $r_{1}=T_{3}-T_{2}, r_{2}=T_{3}-T_{1}, r_{3}=T_{2}-T_{1}$. The monitor readings are values after discretizing each of $r_{1}, r_{2}, r_{3}$ into two bins. Define 4 modes. $N F$ denotes fault free mode, i.e. the normal mode; $F_{1}, F_{2}$ and $F_{3}$ denotes fault in sensor $T_{1}, T_{2}$ and $T_{3}$, respectively. From the monitor functions it is easy to obtain the response matrix as in Table 2. Two datasets are generated by the simulated process, $D_{l}$ and $D_{2}$. Each of them has 100 samples. $D_{l}$ contains 25 samples each from $N F, F_{1}, F_{2}, F_{3}$ while $D_{2}$ includes 50 samples each from $N F$ and $F_{1}$. Besides using the proposed response information-based method, a diagnosis without the background knowledge is also conducted using dataset $D_{2}$.

Table 2 Response matrix in the sample process

\begin{tabular}{lllll}
\hline & $N F$ & $F_{1}$ & $F_{2}$ & $F_{3}$ \\
\hline \hline$r_{1}$ & 0 & 0 & $*$ & $*$ \\
\hline$r_{2}$ & 0 & $*$ & 0 & $*$ \\
\hline$r_{3}$ & 0 & $*$ & $*$ & 0 \\
\hline
\end{tabular}

Table 3 Success diagnosis rates for each mode using different historical datasets with or without the background response information.

\begin{tabular}{lccrr}
\hline & $N F$ & $F_{1}$ & \multicolumn{1}{c}{$F_{2}$} & \multicolumn{1}{c}{$F_{3}$} \\
\hline \hline $\mathrm{D}_{1}: 25$ samples each from $N F, F_{1}, F_{2}, F_{3}$ & $57.0 \%$ & $49.9 \%$ & $58.2 \%$ & $46.3 \%$ \\
\hline $\mathrm{D}_{2}: 50$ samples each from $N F, F_{1}$ & $41.6 \%$ & $38.0 \%$ & $33.3 \%$ & $35.6 \%$ \\
\hline $\mathrm{D}_{2}$ without process knowledge & $87.9 \%$ & $81.9 \%$ & $5.9 \%$ & $5.3 \%$ \\
\hline
\end{tabular}




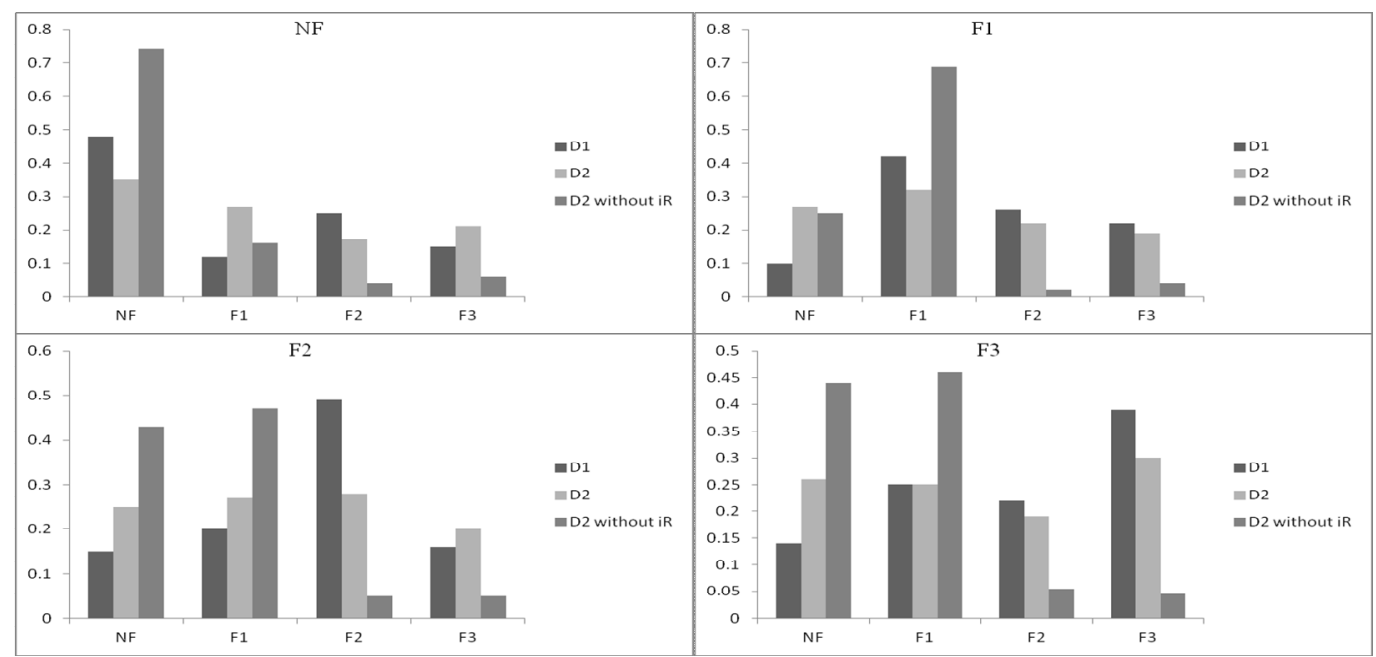

Figure 2 Average probability assigned to each mode. The three colors represent three conditions: using dataset $D_{1}$ and the proposed approach; using $D_{2}$ and the approach; using $D_{2}$ without the approach. The true underlying mode is marked on the top of each graph.

Table 3 shows the success diagnosis rates for each mode using different historical datasets with or without the background response information. And Fig. 2 shows the resulting average probability assigned to each mode. Note that for the four modes only 100 historical samples are used. So if the amount of historical data increases, the diagnosis performance will be even better. For the test using dataset $D_{2}$ without the approach, which is represented by the third bars of each bar group, note that although for underlying mode $N F$ and $F_{1}$, the diagnosis result seems better, it turns out to be wrong when the true mode is $F_{2}$ or $F_{3}$. So its overall performance is not reliable. Also one can see from the middle bars that using the proposed approach even when there is no available historical data from mode $F_{2}$ and $F_{3}$, the approach still yield correct result when the underlying mode is $F_{2}$ or $F_{3}$.

\section{Acknowledgements}

This work was supported by the National Natural Science Foundation of China (Nos. 61304141, 61573296), the Specialized Research Fund for the Doctoral Program of Higher Education of China (No.20130121130004), the Fundamental Research Funds for the Central Universities in China (Xiamen University: Nos. 201412G009, 2014X0217, and 201410384090) and the China Scholarship Council award.

\section{References}

[1] J. J. Gertler, Survey of model-based failure detection and isolation in complex plants, IEEE Control Syst. Mag. vol. 8, no. 6 (1988) 3-11.

[2] V. Venkatasubramanian, R. Rengaswamy, K. Yin, and S.N. Kavuri, A review of process fault detection and diagnosis: Part I: Quantitative model-based methods, Comput. Chem. Eng. vol. 27, no. 3 (2003) 293-311.

[3] V. Venkatasubramanian, R. Rengaswamy, and S.N. Kavuri, A review of process fault detection and diagnosis: Part II: Qualitative models and search strategies, Comput. Chem. Eng. vol. 27, no. 3 (2003) 313-326.

[4] V. Venkatasubramanian, R. Rengaswamy, S.N. Kavuri, and K. Yin, A review of process fault detection and diagnosis: Part III: Process history based methods, Comput. Chem. Eng. vol. 27, no.3 (2003) 327-346.

[5] B. Huang, Bayesian methods for control loop monitoring and diagnosis, J. Process Control. vol. 18, no. 9 (2008) 829-838. 